\title{
। ÖZs-
}

\section{Soziale Gerechtigkeit in der Marktgesellschaft? Zu deren Genese, Bestimmung und sozialpolitischen Durchsetzung}

\section{Workshop am Institut für Soziologie an der Wirtschaftsuniversität Wien,} 31. 3. 2011-2. 4. 2011

Das westeuropäische Sozialstaatsmodell auf Grundlage einer sozial ausgerichteten Marktgesellschaft gerät zunehmend unter Legitimationsdruck: Die Gruppe der ,working poor' ist überall im Anwachsen begriffen, die sozioökonomische Kluft zwischen Teilgruppen innerhalb der Gesellschaften nimmt überall zu, die damit zusammenhängenden gesellschaftlichen Partizipationschancen werden als ungleiche immer offensichtlicher, Diskussionen um Grundsicherung oder Mindesteinkommen erfahren neue Aktualität. Zweifelsohne gehört die diese Phänomene überspannende Frage nach „,sozialer Gerechtigkeit“ zu den umstrittenen „Themen der Zeit“ und allerorten wird sie auch neu verhandelt - wie ,immer schon“ in der Philosophie, immer häufiger in der Soziologie, neuerdings auch in der Ökonomie und im Feld der Politik ohnedies schon längst. Entsprechend groß ist die Variabilität im Verständnis der sozialen Gerechtigkeit, gerade auch im Wandel der Jahre. Dieser Pluralität im Faktischen steht aber nach wie vor der Diskurs um deren Geltungsanspruch gegenüber.

Im Rahmen eines interdisziplinären Workshops soll, ausgehend von zwei rezenten Büchern von Günter Dux - ,, Warum denn Gerechtigkeit. Die Logik des Kapitals. Die Politik im Widerstreit mit der Ökonomie“ (Velbrück 2008) und ,,Von allem Anfang an: Macht, nicht Gerechtigkeit. Studien zur Genese und historischen Entwicklung des Postulats der Gerechtigkeit" (Velbrück 2009) - zu Fragen des gesellschaftstheoretischen, erkenntniskritischen und historischen Kontextes der Genese des modernen Gerechtigkeitsbegriffs, zu dessen gegenwärtigen Verständnissen und Bestimmungen sowie deren sozialpolitischen Umsetzung diskutiert werden.

Als Vortragende konnten u. a. Peter Koller, Stephan Lessenich, Roswitha Pioch, Reinhard Pirker, Volker H. Schmidt und Georg Vobruba gewonnen werden. Erfreulicherweise hat auch Günter Dux seine Teilnahme bereits verbindlich zugesagt, so dass eine direkte Diskussion mit dem Autor ebenfalls möglich sein wird.

An der Thematik Interessierte können, allerdings in zahlenmäßig beschränktem Ausmaß und ohne eigenen Vortrag, am Workshop teilnehmen. Interessentinnen und Interessenten werden gebeten, sich spätestens bis zum 15. Februar 2011 per E-Mail an die Organisatoren zu wenden. Ein kleiner Tagungsbeitrag muss eingehoben werden.

\section{Kontakt:}

Gerda Bohmann, Wirtschaftsuniversität Wien (Gerda.Bohmann@wu.ac.at)

Heinz-Jürgen Niedenzu, Universität Innsbruck (Heinz-Juergen.Niedenzu@uibk.ac.at) 